Mens

revue d'histoire intellectuelle de l'Amérique française

\title{
L'histoire du livre et de l'imprimé au Québec
}

Éric Leroux

Volume 5, numéro 2, printemps 2005

URI : https://id.erudit.org/iderudit/1024372ar

DOI : https://doi.org/10.7202/1024372ar

Aller au sommaire du numéro

Éditeur(s)

Centre de recherche en civilisation canadienne-française

ISSN

1492-8647 (imprimé)

1927-9299 (numérique)

Découvrir la revue

Citer ce document

Leroux, É. (2005). L'histoire du livre et de l'imprimé au Québec. Mens, 5(2),

557-581. https://doi.org/10.7202/1024372ar d'utilisation que vous pouvez consulter en ligne.

https://apropos.erudit.org/fr/usagers/politique-dutilisation/ 


\section{Bibliographie}

\section{L'HISTOIRE DU LIVRE ET DE L'IMPRIMÉ AU QUÉBEC}

Éric Leroux

École de bibliothéconomie et des sciences de l'information Université de Montréal

Depuis quelques années, l'histoire du livre et de l'imprimé a repris du galon au Canada et au Québec. Cette nouvelle vitalité est en partie due aux nombreux chantiers de recherche et d'enseignement qui ont vu le jour dans ce domaine au cours des dernières années. Qu'on pense, par exemple, au projet sur l'Histoire du livre et de l'imprimé au Canada/History of the Book in Canada, à la reprise des journées d'échanges scientifiques de l'Association québécoise pour l'étude de l'imprimé, aux diplômes d'étude créés à l'Université de Sherbrooke (Certificat d'histoire du livre et de l'édition) et au St. Michael's College de la University of Toronto (Book and Media Studies Program), enfin au nouveau programme de soutien à la recherche mis sur pied par la Bibliothèque nationale du Québec.

La recherche en histoire du livre et de l'imprimé, dans son acception canadienne et québécoise, comprend l'ensemble du circuit de l'imprimé : de la production d'un manuscrit à sa distribution, en passant par sa consommation et sa réception. C'est ce circuit que cette bibliographie tente de circonscrire à travers quatre différents thèmes : la lecture, le livre et 
l'imprimé ; la production matérielle du livre ; l'édition; les bibliothèques et les librairies. Nous avons cru bon d'ajouter une section préalable portant sur les principaux outils ou instruments de recherche du domaine. Évidemment, cette bibliographie n'est pas exhaustive : nous avons plutôt sélectionné les titres qui nous semblaient les plus pertinents, ceux qui ont marqué la discipline, ou encore ceux qui ont laissé moins de traces, mais qui méritent qu'on s'y arrête.

Au Québec, nous sommes redevables à Ægidius Fauteux qui fut le premier à nous avoir donné une histoire du livre et de l'imprimerie à la fin des années 1950. Mais au-delà des travaux pionniers de Fauteux, c'est surtout de la France que souffle le vent du changement. Aussi, c'est dans le sillage des travaux de Henri-Jean Martin, de Robert Mandrou et de Roger Chartier que les historiens québécois, Claude Galarneau, Jean-Pierre Wallot et John Hare en tête, publient leurs premières recherches dans le domaine. Depuis, deux ou trois générations de chercheurs s'appliquent à poursuivre le travail de défrichage entrepris par cette première génération de chercheurs en histoire du livre.

Aujourd'hui, nous sommes à l'heure des synthèses et des bilans. À preuve, la publication tant attendue du volume 1 de l'Histoire du livre et de l'imprimé an Canada dirigé par Patricia Fleming, Gilles Gallichan et Yvan Lamonde (2004) et la parution des deux tomes de l'Histoire de l'édition littéraire an Québec au $X X^{e}$ siècle (1999 et 2004), un travail entrepris au début des années 1980 par le Groupe de recherche sur l'édition littéraire au Québec (GRELQ) dirigé par Jacques Michon. Ce n'est pas un hasard non plus si un certain nombre d'éditeurs ont publié leurs mémoires au cours des dernières années (Victor-Lévy Beaulieu, Alain Horic, Jacques Hébert, Jacques Fortin), si Pierre Hébert publiait en 2004 le deuxième tome de sa synthèse sur l'histoire de la censure littéraire au Québec, et si 
le premier bilan de l'histoire de la librairie paraissait au tournant du millénaire, sous la plume de Fernande Roy. Enfin, moment historique parmi d'autres, l'année 2004 marque aussi, pour la première fois, la publication des mémoires d'un bibliothécaire québécois de renom, Jean-Rémi Brault, qui nous offre le récit de son itinéraire professionnel.

Évidemment, le fait de tracer des bilans ne signifie pas qu'il n'y a plus place à de nouvelles recherches et au défrichage de nouvelles avenues pour la jeune (et moins jeune!) génération de chercheurs. En ce sens, cette bibliographie vise également à mettre de l'avant les travaux les plus récents effectués dans le domaine de l'histoire du livre et de l'imprimé au Québec.

\section{Instruments de recherche}

Association québécoise pour l'étude de l'imprimé (AQÉI). Bibliographie des études québécoises sur l'imprimé http://callisto.si.usherb.ca:8080/aqei/bibliographie/ bibliographie.html

AQÉI. Bibliographie des études québécoises sur limprimé, 19701987, Montréal, Bibliothèque nationale du Québec, 1991, 121 p.

AQÉI. Bibliographie des études québécoises sur l'imprimé, 19881989, Montréal, Bibliothèque nationale du Québec, 1993, 73 p.

Beaulieu, André et Jean Hamelin, La presse québécoise des origines à nos jours, 1973 à 1990, Québec, Les Presses de l'Université Laval. Dix tomes.

Bibliographie des manuels scolaires au Québec de Paul Aubin http://www.bibl.ulaval.ca/ress/manscol/ 
Les bibliothèques parlementaires: bistoire, fonctions, services. Bibliographie sélective et annotée par Gilberte Boilard et JeanLuc Fortin, Québec, Direction de la Bibliothèque, 1992, 78 p. (Collection «Bibliographie » $\mathrm{n}^{\circ} 44$ ).

Les bibliothèques parlementaires: histoire, fonctions, services, $1992-$ 2002. Bibliographie sélective par Gilberte Boilard, Québec, Direction de la Bibliothèque, 2002, 17 p. (Collection « Bibliographie » $\mathrm{n}^{\circ} 83$ ).

Bonville, Jean de. La presse québécoise de 1764 à 1917: Bibliographie analytique, Sainte-Foy, Les Presses de l'Université Laval, 1995, 351 p.

Book History on Line

International Bibliography of the History of the Printed Books and Libraries http://www.kb.nl/kb/bho/index2.html

Fleming, Patricia L. et Sandra Alston. Early Canadian Printing. A Supplement to Marie Tremaine's A Bibliography of Canadian Imprints, 1751-1800, Toronto, University of Toronto Press, 1999, 629 p.

Hare, John et Jean-Pierre Wallot, Les imprimés dans le BasCanada, 1801-1810, Montréal, Les Presses de l'Université de Montréal, 1967, 381 p.

History of the Book in Canada/Histoire du livre et de l'imprimé au Canada. Ressources électroniques. http://acsweb2.ucis.dal.ca/hbicdb/index.html

Lamonde, Yvan et Marie-Pierre Turcot. La littérature personnelle au Québec (1980-2000), Montréal, Bibliothèque nationale du Québec, 2000, 100 p. 
Lamonde, Yvan et Claude Beauchamp. Données statistiques sur l'bistoire culturelle $d u$ Québec (1760-1900), Institut interuniversitaire de recherches sur les populations (IREP), 1996, 146 p.

Lemire, Maurice, dir., La vie littéraire au Québec, 1991 à 1999 , Sainte-Foy, Les Presses de l'Université Laval. Quatre tomes. Michon, Jacques, "L'histoire du livre au Québec au XX siècle, orientations bibliographiques ", Présence francophone, 51 (1997), pp. 139-154.

Roy, Fernande et Jean de Bonville. «La recherche sur l'histoire de la presse québécoise. Bilan et perspectives", Recherches sociographiques, XLI, 1 (2000), pp. 15-51.

Statistiques de l'édition au Québec, 1968-1982. Montréal, Bibliothèque nationale du Québec, 1984, 200 p.

Statistiques de l'édition an Québec. Montréal, Bibliothèque nationale du Québec, 1983 à 2002. Publication annuelle. Depuis l'édition de 1998, Statistiques de l'édition au Québec est offert en texte intégral à l'adresse suivante: www.biblinat.gouv.qc.ca

Tremaine, Marie. A Bibliography of Canadian Imprints, 17511800, Toronto, University of Toronto Press, 1952, 750 p.

Vlach, Milada et Yolande Buono. Catalogue collectif des impressions québécoises, 1764-1820, Montréal, Bibliothèque nationale du Québec, 1984, 416 p.

\section{La lecture, le livre et l'imprimé}

Aubin, Paul. Le manuel scolaire dans l'bistoriographie québécoise, Sherbrooke, Ex libris, 1997, 151 p. 
Aubin, Paul. Les communautés religieuses et l'édition du manuel scolaire an Québec, 1765-1964, Sherbrooke, Ex libris, 2001, $131 \mathrm{p}$.

Bernier, Silvie. Du texte à l'image. Le livre illustré au Québec, Sainte-Foy, Les Presses de l'Université Laval, 1990, 335 p.

Brodeur, Raymond. «Le règne d'un livre : le Petit catéchisme $d u$ diocèse de Québec de 1815 », dans Claude Galarneau et Maurice Lemire, dir., Livre et lecture an Québec (1800-1850), Québec, Institut québécois de recherche sur la culture (IQRC), 1988, pp. 149-165.

Buono, Yolande. Imprimerie et diffusion de limprimé à Montréal, de 1776 à 1820, thèse de maîtrise (bibliothéconomie), Université de Montréal, 1980, 216 p.

Cambron, Micheline, dir., Le journal Le Canadien. Littérature, espace public et utopie. 1836-1845, Montréal, Fides, 1999, 419 p.

Fauteux, Ægidius. L'introduction de limprimerie an Canada, Montréal, Compagnie de papier Rolland, 1957, 178 p.

Felteau, Cyrille. Histoire de La Presse, tome 1, 1884-1916, Montréal, Les Éditions La Presse, 1983, 401 p.

Fleming, Patricia L., Gilles Gallichan et Yvan Lamonde, dir., Histoire du livre et de limprimé an Canada. Volume 1, Des débuts à 1840, Montréal, Les Presses de l'Université de Montréal, 2004, 566 p.

Fleming, Patricia L., Cultural Crossroads: Print and Reading in Eighteen- and Nineteenth-Century English-Speaking Montreal, The 2002 James Russell Wiggins Lecture in the History of Book in American Culture at the American Antiquarian Society, Worcester, Massachusetts, American Antiquarian Society, 2004, pp. 231-248. 
Fournier, Alain, Les insolences du frère Untel. Un best-seller de la Révolution tranquille, Québec, Centre de recherche en littérature québécoise, 1988, 159 p.

Gagnon, Jean, « Les livres de récompense et la diffusion de nos auteurs de 1856 à 1931 », Cabiers de bibliologie 1, Québec, 1980, pp. 3-24.

Galarneau, Claude, «Livres et société à Québec (1760-1859). État des recherches ", dans Yvan Lamonde, dir., L'imprimé au Québec. Aspects bistoriques, Québec, IQRC, 1983.

Gallichan, Gilles, Les premiers pas de la lecture publique à Montréal », Documentation et bibliothèques, 41, 3 (1995), pp. 137 142.

Gallichan, Gilles, Livre et politique au Bas-Canada (1791-1849), Sillery, Septentrion. 1991, 519 p.

Gallichan, Gilles, "Les débats parlementaires et la mémoire des mots ", Papers of the Bibliographical Society of Canada/Cabiers de la Société bibliographique du Canada, XXVII (1988), pp. 38-78.

Gallichan, Gilles, "Le livre français au Parlement du BasCanada (1792-1840) ", dans Claude Galarneau et Maurice Lemire, dir., Livre et lecture au Québec (1800-1850), Québec, IQRC, 1988, pp. 115-131.

Gallichan, Gilles, "Bibliothèque et culture après la Conquête", dans Georges-A. Chartrand, dir., Livre, bibliotbèque et culture québécoise, Montréal, vol. 1, ASTED, 1977, pp. 299-310.

Hare, John et Jean-Pierre Wallot, «Le livre au Québec et la librarie Neilson au tournant du XIX siècle", dans Claude Galarneau et Maurice Lemire, dir., Livre et lecture an Québec (1800-1850), Québec, IQRC, 1988, pp. 93-112. 
Hare, John et Jean-Pierre Wallot, «Les imprimés au Québec (1760-1859) ", dans Yvan Lamonde, dir., L'imprimé au Québec: aspects historiques (18-20' siècles), Québec, IQRC, 1983, pp. 77125.

Hébert, Pierre, en collaboration avec Élise Salaün, Censure et littérature au Québec. Des vieux couvents an plaisir de lire, 19201959, Montréal, Fides, 2004, 252 p.

Hébert, Pierre, «Une littérature et ses péchés : dialogue entre l'histoire de la censure et l'histoire des idées aux Québec au $\mathrm{XX}^{\mathrm{e}}$ siècle », dans Damien-Claude Bélanger, Sophie Coupal et Michel Ducharme, dir., Les idées en mouvement: perspectives en histoire intellectuelle et culturelle du Canada, Québec, Les Presses de l'Université Laval, 2004, pp. 211-223.

Hébert, Pierre, "Chant du cygne de la censure cléricale au Québec: la revue Lectures (1946-1966)", Bulletin des bibliothèques de France, 6 (2003), pp. 30-37.

Hébert, Pierre et Patrick Nicol, Censure et littérature au Québec. Le livre crucifié, 1625-1919, Montréal, Fides, 1997, 290 p.

Hébert, Pierre, «La croix et l'ordre : le clergé et la censure de l'imprimé au Québec », Documentation et bibliothèques, 41, 1 (janvier-mars 1995), pp. 21-29.

Kesteman, Jean-Pierre. "Le Progrès» (1874-1878). Étude d'un journal de Sherbrooke, Sherbrooke, Groupe de recherche en histoire des Cantons de l'Est, Université de Sherbrooke, 1979, 204 p.

Lagrave, Jean-Paul de, Fleury Mesplet (1734-1794), imprimeur, éditeur, libraire, joumaliste, Montréal, Patenaude, 1985, 503 p.

Lagrave, Jean-Paul de, Les origines de la presse au Québec (17601791), Montréal, Éditions de Lagrave, 1975, 157 p. 
Lajeunesse, Marcel, "Le livre dans les échanges sulpiciens Paris-Montréal au cours de la première moitié du XIX siècle", dans Claude Galarneau et Maurice Lemire, dir., Livre et lecture au Québec (1800-1850), Québec, IQRC, 1988, pp. 133-147.

Lajeunesse, Marcel, Les sulpiciens et la vie culturelle à Montréal au XIX ${ }^{c}$ siècle, Montréal, Fides, 1982, 278 p.

Lamonde, Yvan, Cultural Crossroads: Print and Reading in Eighteen- and Nineteenth-Century French-Speaking Montreal, The 2002 James Russell Wiggins Lecture in the History of Book in American Culture at the American Antiquarian Society, Worcester, Massachusetts, American Antiquarian Society, 2004, pp. 249-267.

Lamonde, Yvan, "Canadian Print and the Emergence of a Public Culture in the Eighteenth and Nineteenth Centuries", dans Damien-Claude Bélanger, Sophie Coupal et Michel Ducharme, dir., Les idées en mowvement: perspectives en bistoire intellectuelle et culturelle du Canada, Québec, Les Presses de l'Université Laval, 2004, pp. 175-189.

Lamonde, Yvan et Sophie Montreuil, "Pour une histoire des pratiques de lecture: éléments de méthode et pacte fondateur ", dans Yvan Lamonde et Sophie Montreuil, dir., Lire au Québec au XIX ${ }^{e}$ siècle, Saint-Laurent, Fides, 2003, pp. 716.

Landry, Kenneth, "Des Reader's Digest pour un public lettré : le magazine et le recueil littéraire canadien avant 1840 », 7 p. http://www.library.utoronto.ca/hbic/vol1landry_en.html

Lebel, Jean-Marie, «La presse quotidienne de Québec en 1900, à une croisée de siècles et de mondes ", dans Yves Roby et Nive Voisine, dir., Érudition, humanisme et savoir. Actes du colloque en l'honneur de Jean Hamelin, Sainte-Foy, Presses de l'Université Laval, 1996, pp. 377-400. 
Lemire, Maurice, «Romans-feuilletons et extraits littéraires dans les journaux canadiens de 1830 à 1850 », dans Claude Galarneau et Maurice Lemire, dir., Livre et lecture an Québec (1800-1850), Québec, IQRC, 1988, pp. 183-194.

Lemoine, Réjean, "Le commerce du livre et la lecture à Québec avant 1837», dans Yvan Lamonde et Gilles Gallichan, dir., L'bistoire de la culture et de l'imprimé. Hommages à Claude Galarneau, Québec, Presses de l'Université Laval, 1996, pp. 163-172.

Marquis, Dominique, Un quotidien pour l'Église. L'Action catholique, 1910-1940. Montréal, Leméac, 2004, 220 p.

Mativat, Daniel, Le métier d'écrivain au Québec (1840-1900): pionniers, nègres ou épiciers des lettres?, Montréal, Tryptique, 1996, 510 p.

Melançon, Benoit, dir., Le savoir des livres, Montréal, Les Presses de l'Université de Montréal, 2005.

Melançon, François, «La circulation du livre au Canada sous la domination française ", Papers of the Bibliographical Society of Canada/Cabiers de la Société bibliographique du Canada, 37, 2 (automne 1999), pp. 35-58.

Melançon, François, «Façonner et surveiller l'intime : lire en Nouvelle-France ", dans Manon Brunet et Serge Gagnon, dir., Discours et pratiques de l'intime, Québec, IQRC, 1993, pp. 17-45.

Montreuil, Sophie, «L'CEuvre des livres gratuits (1898-1908) dans l'histoire de la lecture publique au Québec", Documentation et bibliothèques, 49, 3 (juillet-septembre 2003), pp. 129-135.

Montreuil, Sophie, «(Se) lire et (se) dire: Joséphine Marchand-Dandurand et la lecture (1879-1886) », dans Yvan Lamonde et Sophie Montreuil, dir., Lire au Québec au XIX è siècle, Saint-Laurent, Fides, 2003, pp. 123-150. 
Olivier, Daniel, Philéas Gagnon, bibliophile, thèse de maitrise (bibliothéconomie), Université de Montréal, 1978, 96 p.

Panneton, Jacques, «La lecture publique au Québec de 1959 à demain", Documentation et bibliothèques, 41, 3 (juilletseptembre 1995), pp. 153-158.

Parent, Françoise, «Les envois de livres de Paris au BasCanada, de 1824 à 1827 ", dans Claude Galarneau et Maurice Lemire, dir., Livre et lecture au Québec (1800-1850), Québec, IQRC, 1988, pp. 29-42.

Parker, George L., The Beginnings of the Book Trade in Canada, Toronto, University of Toronto Press, 1985, 346 p.

Robert, Lucie, "Prolégomènes à une étude sur les transformations du marché du livre au Québec (1900-1940)", dans Yvan Lamonde, dir., Limprimé au Québec: aspects historiques (18'-20 siècles), Québec, IQRC, 1983, pp. 225-242.

Robert, Mario, "Le livre et la lecture dans la noblesse canadienne, 1670-1764», Revue d'bistoire de l'Amérique française, 56, 1 (été 2002), pp. 3-27.

Robert, Mario, Le livre et la lecture dans la noblesse canadienne, 1670-1764, thèse de maitrise (histoire), Université du Québec à Montréal, 2000, 165 p.

Roy, Fernande, «Les intellectuels canadiens-français du siècle dernier: les journalistes ", dans Manon Brunet et Pierre Lanthier, dir., Linscription sociale de l'intellectuel, Sainte-Foy/ Paris, Les Presses de l'Université Laval/L'Harmattan, 2000, pp. 341-350.

St-Jacques, Denis, et al., Ces livres que vous avez aimés. Les bestsellers au Québec de 1970 à aujourd'bui, Québec, Nuit blanche, 1997, $350 \mathrm{p}$. 
Vincent, Josée, Les professionnels du livre à la conquête de leur marché: les associations professionnelles dans le champ littéraire au Québec (1921-1960), thèse de doctorat (lettres et communications), Université de Sherbrooke, 2002, 370 p.

Vincent, Josée, "Les salons du livre à Montréal, ou quand "livre" rime avec...", dans Josée Vincent et Nathalie Watteyne, dir., Autour de la lecture. Médiations et communautés littéraires, Québec, Éditions Nota bene, 2002, pp. 207-228.

Vincent, Josée, Les tribulations du livre québécois en France (1959. 1985), Québec, Nuit Blanche Éditeur, 1997, 230 p.

Vincent, Josée, «Le Conseil supérieur du livre : pour un statut du livre au Québec ", dans Jacques Michon, dir., Édition et pouvoirs, Sainte-Foy, Les Presses de l'Université Laval, 1995, pp. 205-216.

Vincent, Josée, «Le Conseil supérieur du livre, du rapport Bouchard à la loi 51 : pour une politique du livre au Québec ", Présence francophone, 45 (1994), pp. 173-191.

Vincent, Sophie, L'apprentissage de la liberté. Mutations de la censure au Québec, de l'abolition de l'Index aux lendemains de la crise d'Octobre (1966-1971), thèse de maitrise (lettres et communications), Université de Sherbrooke, 2002, 186 p.

Yergeau, Robert, Art, argent, arrangement. Le mécénat d'État, Ottawa, Les éditions David, 2004, 632 p.

\section{La production matérielle du livre et de l'imprimé}

Belzile, Yves, «La grève des typographes de Montréal (19211924) ", Cabiers d'bistoire, 11, 2 (août 1990), pp. 47-71.

Bonville, Jean de, La presse québécoise de 1884 à 1914. Genèse d'un mass média, Sainte-Foy, Les Presses de l'Université Laval, 1988, 416 p. 
Bourdon, Joseph, Montréal-Matin. Son histoire, ses histoires, Montréal, Les Éditions La Presse, 1978, 282 p.

Chevrefils, Yves, «John Henry Walker (1831-1899), artisangraveur ", The Journal of Canadian Art History/Annales d'bistoire de l'art canadien, 8, 2 (1985), pp. 178-224.

Dansereau, Bernard, «La fabrication des caractères d'imprimerie à Montréal au milieu du XIX ${ }^{e}$ siècle", Revue d'bistoire de l'Amérique française, 47, 1 (été 1993), pp. 83-92.

Dansereau, Bernard, L'avènement de la linotype : le cas de Montréal à la fin du XIX' siècle, Montréal, VLB éditeur, 1992, $150 \mathrm{p}$.

Demers, François, Communications et syndicalisme : des imprimeurs aux journalistes, Montréal, Méridien, 1989, 203 p.

Desrochers, Luc, «Les facteurs d'apparition du syndicalisme catholique dans l'imprimerie, 1921-1945", Revue d'bistoire de l'Amérique française, 37 (septembre 1983), pp. 241-269.

Devost, Alain, L'imprimerie an Québec, Québec, Gouvernement du Québec, Commission de la santé et de la sécurité du travail, 1982, 366 p.

Dewalt, Bryan, Technology and Canadian Printing: $A$ History from Lead Type to Lasers, Ottawa, National Museum of Science and Technology, 1995. $161 \mathrm{p}$.

Galarneau, Claude, "Le premier siècle de l'imprimé au Québec (1764-1870)", dans Jacques Michon et Jean-Yves Mollier, dir., Les mutations du livre et de l'édition dans le monde, Sainte-Foy/Paris, PUL/L'Harmattan, 2001, pp. 80-83.

Galarneau, Claude, "Les Desbarats: une dynastie d'imprimeurs-éditeurs (1794-1893) ", Les Cabiers des Dix, 46 (1991), pp. 125-149. 
Galarneau, Claude, "Les métiers du livre à Québec», Les Cabiers des Dix, 43 (1983), pp. 143-165.

Grosbois, Guy de, «Le métier de relieur », Le Maître imprimeur, 60, 8 (août 1996), pp. 25-26; 61, 3 (mars 1997), pp. 19-20; 61, 8 (août 1997), p. 19; 62, 4 (avril 1998), p. $21 ; 62,9$ (octobre 1998), p. 17.

Grosbois, Guy de, «Pour une histoire de la reliure québécoise : matériaux et perspectives de recherche", Cinquième forum international de la reliure d'art, Montréal, Les Amis de la reliure d'art, ARA Canada, 1996, pp. 25-31.

Hill, Robert A., Robert Sellar and The Huntingdon Gleaner. Voice of the Vanishing Minority, 1863-1919, Montréal, McGillQueen's University Press, 1998, 392 p.

Lapointe, Raoul, Histoire de limprimerie an Saguenay (18791969), Chicoutimi, La Société historique du Saguenay, 1969, $292 \mathrm{p}$.

Lebel, Jean-Marie, "Imprimer à Québec au XIX siècle : l'œuvre de Joseph "C" Darveau, typographe", dans Yvan Lamonde et Gilles Gallichan, dir., L'bistoire de la culture et de limprimé. Hommages à Claude Galarneau, Sainte-Foy, Presses de l'Université Laval, 1996, pp. 189-206.

Leduc, Jean, Le livre matériel de poésie au Québec de 1950 à 1970, thèse de maitrise (bibliothéconomie), Université de Montréal, 1980, 169 p.

Leroux, Éric, «Culture ouvrière et métiers du livre : la Société typographique de Québec, 1836-1872», Papers of the Bibliographical Society of Canada/Cabiers de la Societé bibliographique du Canada, 42, 2 (automne 2004), pp. 25-56.

Thibault, Benoit, L'imprimerie Gawin, 1892-1970: première imprimerie commerciale de la ville de Hull (Québec), Magog, Association des Gauvin d'Amérique, 1999, 31 p. 
Vincent, Paul, L'imprimerie coopérative Harpell: ses origines exceptionnelles, son développement, Montréal, Chaire de coopération Guy-Bernier de l'UQAM, 1996, 56 p.

\section{L'édition}

Beaudry, Jacques, «Le commerce de l'édition : Fernand Pilon, libraire-éditeur», dans Groupe de recherche sur l'édition littéraire au Québec (GRELQ), Éditeurs transatlantiques, Sherbrooke/Montréal, Ex libris/Tryptique, 1991, pp. 81-129.

Beaulieu, Victor-Lévy, Les mots des autres. La passion d'éditer, Montréal, VLB éditeur, 2001, 237 p.

Béland, Caroline, «Les Éditions Mille Roches (1976-1989) : une mission régionale", Mens. Revue d'bistoire intellectuelle de l'Amérique française, II, 2 (printemps 2002), pp. 233-259.

Bergeron, Liette, "Les Éditions du Totem (1933-1938)», dans Jacques Michon, dir., L'édition littéraire en quête d'autonomie. Albert Lévesque et son temps, Sainte-Foy, Les Presses de l'Université Laval, 1994, pp. 41-56.

Bernier, Silvie, «Un résistant canadien: l'éditeur Lucien Parizeau ", dans Groupe de recherche sur l'édition littéraire au Québec (GRELQ), Éditeurs transatlantiques, Sherbrooke/ Montréal, Ex libris/Tryptique, 1991, pp. 43-79.

Bernier, Silvie, "Bernard Valiquette. La nouvelle image de l'édition québécoise ", dans Louis Chantigny et Silvie Bernier, dir. Bernard Valiquette, Ottawa, Publie-Liaison, 1986, pp. 6781.

Boisclair, Isabelle, «Les femmes éditrices au Québec : repères historiques ", Présence francophone, 47 (1995), pp. 151-167.

Cau, Ignace, L'édition au Québec de 1960 à 1977, Québec, ministère des Affaires Culturelles, 1981, 229 p. 
Chalifoux, Jean-Pierre, L'édition au Québec, 1940-1950, thèse de maitrise (bibliothéconomie), Université de Montréal, 1973, $105 \mathrm{p}$.

Chantigny, Louis et Silvie Bernier, dir., Bernard Valiquette, Ottawa, Publie-Liaison, 1986, 109 p.

Cloutier, Yvan, «L'incursion des communautés religieuses dans l'édition littéraire: les franciscains, les dominicains, les frères et les pères de la Congrégation de Sainte-Croix ", Papers of the Bibliographical Society of Canada/Cabiers de la Societé bibliographique du Canada, 32, 2 (automne 1994), pp. 121-133.

Cloutier, Yvan, "L'activité éditoriale des dominicains: les Éditions du Lévrier (1937-1975) ", dans Jacques Michon, dir., L'édition littéraire en quête d'autonomie. Albert Lévesque et son temps, Sainte-Foy, Les Presses de l'Université Laval, 1994, pp. 7797.

Cloutier, Yvan, «Du prêche et de l'imprimé : hégémonie et orthodoxie. L'Église catholique face à l'imprimé », dans Jacques Michon, dir., Édition et pouvoirs, Sainte-Foy, Les Presses de l'Université Laval, 1995, pp. 89-97.

Faure, Sylvie, « Pouvoirs politiques et stratégies éditoriales au Québec (1960-1990)», dans Jacques Michon, dir., Édition et pouvoirs, Sainte-Foy, Les Presses de l'Université Laval, 1995, pp. 193-203.

Faure, Sylvie, "Le roman sentimental et la biographie romancée: les Éditions de l'Étoile», dans Groupe de recherche sur l'édition littéraire au Québec (GRELQ), L'édition du livre populaire, Sherbrooke, Ex libris, 1988, pp. 111131.

Fortin, Jacques, L'aventure: récit d'un éditeur, Montréal, Québec/Amérique, 2000, 284 p. 
Gallichan, Gilles, "L'édition gouvernementale au Québec depuis le $18^{e}$ siècle ", dans Yvan Lamonde, dir., L'imprimé au Québec: aspects historiques (18`-20 siècles), Québec, IQRC, 1983, pp. 262-287.

Giguère, Richard, "Un surréalisme sans frontières: les Éditions Erta», dans Groupe de recherche sur l'édition littéraire au Québec (GRELQ), L'édition de poésie, Sherbrooke, Ex libris, 1989, pp. 55-86.

Giguère, Richard, "Une aventure de typographie: des arts graphiques aux Éditions Erta », Études françaises, 18 (automne 1982), pp. 99-104.

Guay, Jean-Pierre, Lorsque notre littérature était jeune. Entretiens avec Pierre Tisseyre, Montréal, Cercle du livre de France, 1983, 264 p.

Hébert, Jacques, En 13 points Garamond, Trois-Pistoles, Éditions Trois-Pistoles, 2002, 143 p.

Hamelin, Carole, «Deux femmes éditrices: les Éditions Quartz ", dans Groupe de recherche sur l'édition littéraire au Québec (GRELQ), L'édition de poésie, Sherbrooke, Ex libris, 1989, pp. 145-161.

Horic, Alain, Mon parcours d'éditeur avec Gaston Miron, Montréal, L'Hexagone, 2004, 173 p.

Janelle, Claude, Les éditions du Jour. Une génération d'écrivains, Montréal, Hurtubise HMH, 1983, 333 p.

Landry, François, Beauchemin et l'édition au Québec. Une culture modèle, 1840-1940, Montréal, Fides, 1997, 367 p.

Landry, François, «Les Éditions Édouard Garand et les années 20 », dans Groupe de recherche sur l'édition littéraire au Québec (GRELQ), L'édition du livre populaire, Sherbrooke, Ex libris, 1988, pp. 35-76. 
Landry, François, «Un imprimeur régional: les Éditions Marquis ", dans Groupe de recherche sur l'édition littéraire au Québec (GRELQ), L'édition du livre populaire, Sherbrooke, Ex libris, 1988, pp. 133-151.

Lemire, Maurice, "Les relations entre écrivains et éditeurs au Québec au 19e siècle », dans Yvan Lamonde, dir., L'imprimé au Québec: aspects bistoriques (18-20 siècles), Québec, IQRC, 1983, pp. 207-224.

Lévesque, Claire, "Albert Lévesque, mon père ", dans Jacques Michon, dir., L'édition littéraire en quête d'autonomie. Albert Lévesque et son temps, Sainte-Foy, Les Presses de l'Université Laval, 1994, pp. 131-163.

Luneau, Marie-Pier, Lionel Groulx. Le mythe du berger, Montréal, Leméac, 2003, 226 p.

Luneau, Marie-Pier, "L'effet pseudonyme», dans Josée Vincent et Nathalie Watteyne, dir., Autour de la lecture. Médiations et communautés littéraires, Québec, Éditions Nota bene, 2002, pp. 13-23.

Marquis, André, "Conscience politique et ouverture culturelle : les Éditions d'Orphée ", dans Groupe de recherche sur l'édition littéraire au Québec (GRELQ), L'édition de poésie, Sherbrooke, Ex libris, 1989, pp. 87-120.

Martin, Annie, L'imprimerie L'Action sociale Ltée: l'Église catholique dans l'arène de l'édition québécoise, 1907-1939, thèse de maîtrise (histoire), Université du Québec à Montréal, 2003, $149 \mathrm{p}$.

Michaud, Paul, $A$ u temps de l'Index. Mémoires d'un éditeur, 1949 1961, Montréal, Libre Expression, 1996, 283 p.

Michon, Jacques, dir., Histoire de l'édition littéraire au Québec au $X X^{e}$ siècle. Volume 2: Le temps des éditeurs, 1940-1959, Montréal, Fides, 2004, 533 p. 
Michon, Jacques, dir., Histoire de l'édition littéraire au Québec an $X X^{e}$ siècle. Volume 1: La naissance de l'éditeur, 1900-1939, Montréal, Fides, 1999, 482 p.

Michon, Jacques, "Édition et lecture », dans Josée Vincent et Nathalie Watteyne, dir., Autour de la lecture. Médiations et communautés littéraires, Québec, Éditions Nota bene, 2002, pp. 319-329.

Michon, Jacques et Jean-Yves Mollier, dir., Les mutations $d u$ livre et de l'édition dans le monde, du XVIII siècle à l'an 2000, Québec/Paris, Les Presses de l'Université Laval/L'Harmat$\tan , 2001,597$ p.

Michon, Jacques, Fides. La grande aventure éditoriale du père PaulAimé Martin, Montréal, Fides, 1998, 387 p.

Michon, Jacques, "Industries du livre et mutation du champ éditorial au $\mathrm{XX}^{\mathrm{e}}$ siècle : l'État et l'édition au Canada ", dans Jacques Michon, dir., Édition et pouvoirs, Sainte-Foy, Les Presses de l'Université Laval, 1995, pp. 35-44.

Michon, Jacques, "Albert Lévesque, entre "individualistes" et nationalistes", dans Jacques Michon, dir., L'édition littéraire en quête d'autonomie. Albert Lévesque et son temps, Sainte-Foy, Les Presses de l'Université Laval, 1994, pp. 101-114.

Michon, Jacques, «Les Éditions de l'Arbre (1941-1948)», dans Groupe de recherche sur l'édition littéraire au Québec (GRELQ), Éditeurs transatlantiques, Sherbrooke/Montréal, Ex libris/Tryptique, 1991, pp. 13-41.

Normand, Sylvio, «La contrefaçon d'ouvrages juridiques au Québec au XIX ${ }^{e}$ siècle », dans Jacques Michon, dir., Édition et pouvoirs, Sainte-Foy, Les Presses de l'Université Laval, 1995, pp. 65-79. 
Normand, Sylvio, « Une lignée d'éditeurs-libraires montréalais, spécialistes en droit, au tournant du siècle », Cabiers de la Société bibliographique du Canada, XXXI, 1 (printemps 1993), pp. 7 55.

Parent, Mario, «Développement de l'auto-édition littéraire au Québec: la lutte pour la reconnaissance», dans Jacques Michon, dir., Édition et powvoirs, Sainte-Foy, Les Presses de l'Université Laval, 1995, pp. 99-111.

Parent, Mario, «Les Éditions Serge Brousseau et les Éditions Mangin ", dans Groupe de recherche sur l'édition littéraire au Québec (GRELQ), Éditeurs transatlantiques, Sherbrooke/ Montréal, Ex libris/Tryptique, 1991, pp. 131-159.

Saint-Germain, Richard, «Pirates et contrebandiers dans l'édition populaire au Québec (1940-1960)", dans Jacques Michon, dir., Édition et powvoirs, Sainte-Foy, Les Presses de l'Université Laval, 1995, pp. 81-88.

Spadoni, Carl, "The Publishers Press of Montreal », Papers of the Bibliographical Society of Canada/Cabiers de la Societé bibliographique du Canada, XXIV, (1985), pp. 38-50.

Stanké, Alain, Occasions de bonbeur, Montréal, Stanké, 1993, $489 \mathrm{p}$.

Tellier, Christine, Jeunesse et poésie : de l'Ordre de Bon temps aux Éditions de l'Hexagone, Saint-Laurent, Fides, 2003, 332 p.

Tisseyre, Pierre, "L'édition au Québec », dans L'évolution du rôle social de limprimé et de ses agents au Québec, Montréal, Les conférences Ægidius-Fauteux, 1980, pp. 1-19.

Turgeon, Robert, Analyse du marché de l'édition de livres au Québec, Montréal, Hautes Études commerciales, 1984, 154 p.

Vaugeois, Denis, L'amour du livre. L'édition au Québec, ses petits secrets et ses mystères, Sillery, Septentrion, 2005, 224 p. 


\section{Les bibliothèques et les librairies}

Baillargeon, Jean-Paul, "La littérature dans les menus de lecture des usagers de la bibliothèque de la ville de Québec ", dans Josée Vincent et Nathalie Watteyne, dir., Autour de la lecture. Médiations et communautés littéraires, Québec, Éditions Nota bene, 2002, pp. 267-282.

Baillargeon, Jean-Paul, "Les libraires et les bibliothèques publiques ", dans Denise Lemieux, dir., Traité de la culture, Sainte-Foy, Les éditions de l'IQRC, 2002, pp. 529-537.

Baillargeon, Jean-Paul, "Les bibliothèques publiques du Québec, 1961-1989», Documentation et bibliothèques, (juilletseptembre 1992), pp. 139-147.

Brault, Jean-Rémi, Regard sur l'évolution des bibliothèques québécoises: récit d'un itinéraire professionnel, Montréal, Les Éditions ASTED, 2004, 282 p.

Brisson, Frédéric, «Les librairies et leur impact sur la lecture », dans Josée Vincent et Nathalie Watteyne, dir., Autour de la lecture. Médiations et communautés littéraires, Québec, Éditions Nota bene, 2002, pp. 251-265.

Dagenais, Michèle, «Vie culturelle et pouvoirs publics locaux. La fondation de la bibliothèque municipale de Montréal», Urban History/Revue d'bistoire urbaine, 24, 2, pp. 40-56.

Déry, Boris, La librairie Rolland, 1842-1888, thèse de maitrise (histoire), Université du Québec à Montréal, 2003, 188 p.

Ducharme, Isabelle, "L'offre de titres littéraires dans les catalogues de bibliothèques de collectivités de Montréal (1797-1898), dans Yvan Lamonde et Sophie Montreuil, dir., Lire au Québec au XIX' siècle, Saint-Laurent, Fides, 2003, pp. 237-277. 
Galarneau, Claude, "Clergé, bourgeoisie et lecture publique. La bibliothèque paroissiale de Notre-Dame de Québec ", Les Cahiers des Dix, 54 (2000), pp. 99-117.

Gallichan, Gilles, dir., Au fil des pages et du temps... La Bibliothèque de l'Assemblée nationale, deux siècles d'bistoire, Québec, Bibliothèque de l'Assemblée nationale, 2002, 122 p.

Gallichan, Gilles et Maurice Pellerin, Pamphile Le May, écrivain et bibliothécaire, Montréal, Bibliothèque nationale du Québec, 1987, 147 p.

Garand, Dominique, "La librairie et la distribution: Granger Frères ", dans Groupe de recherche sur l'édition littéraire au Québec (GRELQ), L'édition du livre populaire, Sherbrooke, Ex libris, 1988, pp. 153-186.

Hanson, Elizabeth Ida, A Jewel in the Park: Westmount Public Library, 1897-1918, Montréal, Véhicule Press, 1997, 153 p.

Harvey, Fernand, «Les bibliothèques publiques et la société du savoir", dans Jean-Paul Baillargeon, dir., Bibliothèques publiques et transmission de la culture à l'orée du XXI siècle, Montréal, Les Éditions ASTED/Les Éditions de I'IQRC, 2004, pp. 49-60.

Jenkins, Phil, Une bibliothèque vivante : l'bistoire tant attendue de la Bibliothèque publique d'Ottawa, 1906-2001, Ottawa, Bibliothèque publique d'Ottawa, 2002, 168 p.

Lajeunesse, Marcel, Lecture publique et culture au Québec, SaintFoy, Les Presses de l'Université du Québec, 2004, 228 p.

Lajeunesse, Marcel, «La bibliothèque publique au Québec de la Révolution tranquille au $\mathrm{XIX}^{\mathrm{e}}$ siècle: les acquis et les défis ", dans Jean-Paul Baillargeon, dir., Bibliothèques publiques et transmission de la culture à l'orée du XXI siècle, Montréal, Les Éditions ASTED/Les Éditions de l'IQRC, 2004, pp. 35-47. 
Lajeunesse, Marcel, «Les bibliothèques paroissiales, précurseurs des bibliothèques publiques au Québec?", dans Les bibliothèques québécoises d'hier à aujourd'bui, Montréal, Les Éditions ASTED, 1998, pp. 43-66.

Lajeunesse, Marcel, "L'évolution des bibliothèques publiques du Québec vue par les études et les rapports ", dans Paule Rolland-Thomas, dir., Prospectives en bibliothéconomie et en sciences de linformation. Hommages à Laurent-G. Denis, Montréal, ASTED, 1990, pp. 113-132.

Lajeunesse, Marcel, "La lecture publique au Québec au XX siècle. L'ambivalence des solutions", dans Yvan Lamonde, dir., L'imprimé au Québec: aspects bistoriques (18-20 siècles), Québec, IQRC, 1983, pp. 191-205.

Lajeunesse, Marcel, «Les Bibliothèques publiques à Montréal au début du $\mathrm{XX}^{\mathrm{e}}$ siècle: essai d'histoire socio-culturelle", dans Peter McNally, dir., Readings in Canadian Library History, Ottawa, Canadian Library Association, 1996, pp. 173-198.

Lamonde, Yvan, "Une contribution à l'histoire de la bibliothèque publique au XIX ${ }^{\mathrm{e}}$ siècle", dans Les bibliothèques québécoises d'bier à aujourd'bui, Montréal, Les Éditions ASTED, 1998, pp. 21-28.

Lamonde, Yvan, La librairie et l'édition à Montréal, 1776-1920, Montréal, Bibliothèque nationale du Québec, 1991, 198 p.

Lamonde, Yvan, «La bibliothèque de l'Institut canadien de Montréal, 1852-1876 : pour une analyse multidimensionnelle », dans Yvan Lamonde, Territoires de la culture québécoise, SainteFoy, Les Presses de l'Université Laval, 1991, pp. 117-148.

Lamonde, Yvan, Les bibliothèques de collectivités à Montréal (17'19 siècles), Montréal, ministère des Affaires culturelles, BNQ, 1979, 139 p. 
Lassonde, Jean-René, La Bibliothèque Saint-Sulpice, 1910-1931, Montréal, Bibliothèque nationale du Québec, 1987, 400 p.

Monette, Isabelle, "L'offre de titres littéraires dans les catalogues de la librairie montréalaise (1816-1879)», dans Yvan Lamonde et Sophie Montreuil, dir., Lire an Québec an $X I X^{e}$ siècle, Saint-Laurent, Fides, 2003, pp. 201-235.

Morin, Yvan, «Les bibliothèques privées à Québec d'après les inventaires après décès (1800-1819) », dans Yvan Lamonde, dir., Limprimé au Québec: aspects historiques (18 ${ }^{e}-20^{\circ}$ siècles), Québec, IQRC, 1983, pp. 145-165.

Payeur, Jean, "La bibliothèque publique: lieu d'accès à la connaissance, à la culture et à l'information ", dans Politique culturelle et bibliothèque publique. Lien de diffusion des savoirs, Montréal, ASTED, 2002, pp. 87-106.

Roy, Fernande, Histoire de la librairie an Québec, Montréal, Leméac, 2000, 232 p.

Roy, Jean-Louis, "La librairie Crémazie», dans Réjean Robidoux et Paul Wyczynski, dir., Crémazie et Nelligan, recueil d'etudes, Montréal, Fides 1981, pp. 11-43.

Roy, Jean-Louis, "Une région culturelle mal connue: le pouvoir des librairies ou les libraires du pouvoir», dans L'évolution du rôle social de limprimé et de ses agents au Québec, Montréal, Les conférences Ægidius-Fauteux, 1980, pp. 23-43.

Roy, Jean-Louis, Édouard-Raymond Fabre, libraire et patriote canadien (1799-1854): contre lisolement et la sujétion, Montréal, Hurtubise HMH, 1974, 220 p.

Savard, Réjean, «L'évolution des bibliothèques publiques au Québec de 1960 à 1995 ", dans Les bibliothèques québécoises d'bier à aujourd'bui, Montréal, Les Éditions ASTED, 1998, pp. 143-160. 
Savard, Réjean, "Le discours sur la lecture et l'évolution des bibliothèques publiques au Québec de 1850 à 1950 », Argus, 26, 2 (automne 1997), pp. 19-27.

Tranquille, Henri, Entretiens sur la passion de lire. Henri Tranquille interviewé par Yves Beauchemin, Montréal, Québec/Amérique, 1993, 291 p. 